\title{
The Regge-plus-resonance model for kaon production on the proton and the neutron
}

\author{
J. Ryckebusch, L. De Cruz, P. Vancraeyveld and T. Vrancx \\ Department of Physics and Astronomy, Ghent University, Belgium
}

\begin{abstract}
The Regge-plus-resonance (RPR) framework for kaon photoproduction on the proton and the neutron is an economical single-channel model with very few parameters. Not only does the RPR model allow one to extract resonance information from the data, it has predictive power. As an example we show that the RPR model makes fair predictions for the $p\left(e, e^{\prime} K^{+}\right) \Lambda$ and the $n\left(\gamma, K^{+}\right) \Sigma^{-}$observables starting from amplitudes optimized for the reaction $p\left(\gamma, K^{+}\right) \Lambda$ and $p\left(\gamma, K^{+}\right) \Sigma^{0}$ respectively.
\end{abstract}

Keywords: Kaon photoproduction, Regge phenomenology, nucleon resonances

PACS: 11.55.Jy, 12.40.Nn, 13.60.Le, 14.20.Gk

\section{INTRODUCTION}

These are exciting times for groups working on the modeling of photoinduced open strangeness production on the nucleon $N(\gamma, K) Y$. Indeed, over the last couple of years high-quality data in an extended energy range have become available. The data include single and double polarization observables. The selfanalyzing character of the $\Lambda$ facilitates so-called complete measurements for the $p\left(\gamma, K^{+}\right) \Lambda$ process. These measurements could provide a way to determine the four independent amplitudes for kaon photoproduction at various combinations of the kinematic variables $(s, t)$. Modeling of $N(\gamma, K) Y$ reactions poses some real challenges which stem from the fact that one is dealing with a weak channel (with cross sections of the order of $\mu b$ ) in an energy range where one expects many overlapping resonances. Accordingly, the background-resonance separation is even more challenging than in the pion production channels.

Coupled-channel approaches provide an analysis framework which are very demanding on human resources and involve a large amount of parameters. They represent tools to analyze photomeson production data in the search of $N^{*}$ and $\Delta^{*}$ information like masses, widths and transition form factors, The Regge-plus-resonance (RPR) framework for $N(\gamma, K) Y$ and $N\left(e, e^{\prime} K\right) Y$ developed by the Ghent group [1,2] is an economical single-channel model with very few parameters. The RPR model is sufficiently simple so that it can be used as elementary production operator in hypernuclear calculations for example. In this contribution, we illustrate that the RPR model has predictive power. First, one can reasonably predict $p\left(e, e^{\prime} K^{+}\right) \Lambda$ observables from amplitudes which are optimized against the $p\left(\gamma, K^{+}\right) \Lambda$ data. Second, fair results for "neutron" targets are obtained by appropriately transforming the amplitudes for kaon photoproduction on the proton. 


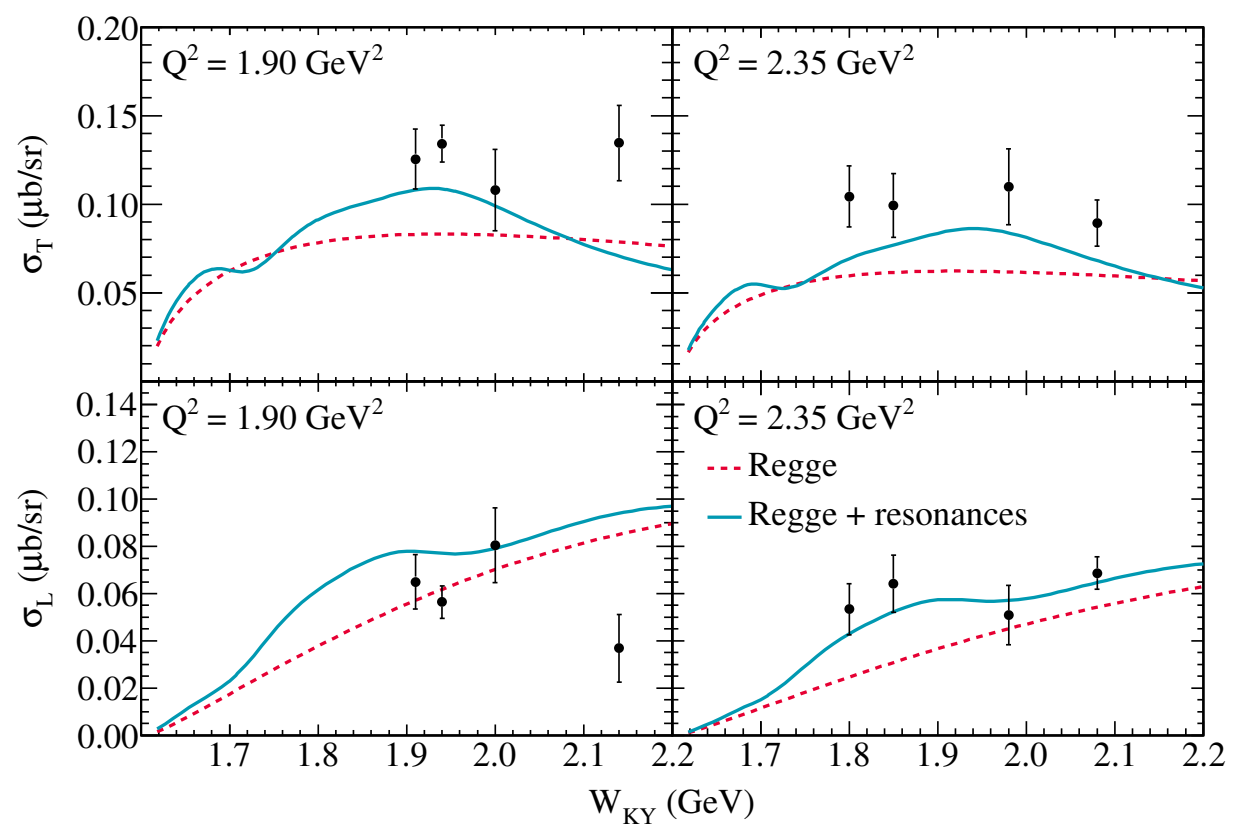

FIGURE 1. Transverse and longitudinal $p\left(e, e^{\prime} K^{+}\right) \Lambda$ cross sections as a function of the kaon-hyperon invariant mass $W_{K Y}$ for $\cos \theta_{K}^{*} \approx 1$ and two values of the four-momentum transfer $Q^{2}$. The dashed lines show the computed contribution from the background diagrams. The solid lines include the background and resonant contributions. The data are from [3].

\section{THE RPR MODEL: LESS CAN BE MORE}

In the RPR model for $\gamma+p \longrightarrow K^{+}+\Lambda$ the background is described by reggeizing the Feynman diagrams involving pseudoscalar $K^{+}(494)$ and vector $K^{*+}(892)$ meson exchange in the $t$ channel. This results in a mere three parameters which fully determine the background [4]. Adding the electric part of a reggeized s-channel Born diagram ensures that one obtains a gauge-invariant model [5]. In the RPR-2007 version of the model the background parameters are optimized against the $p\left(\gamma, K^{+}\right) \Lambda$ data for $E_{\gamma} \geq$ $5 \mathrm{GeV}$. These data were obtained in the sixties and seventies, and it has been pointed out recently that there may be some normalization issues [6]. The RPR-2011 version of the model uses the high-energy part of the recent Jefferson Lab data of Ref. [7] to optimize the background parameters. The resonance contributions are added to the reggeized background at the amplitude level. The resonances constitute the $s$ channel and are treated in the standard fashion with Feynman diagrams. The RPR-2007 model considers the contribution of the $S_{11}(1650), P_{11}(1710), P_{13}(1720), P_{13}(1900)$, and $D_{13}(1900)$ resonances to $\gamma+p \longrightarrow K^{+}+\Lambda$. Since 2007 more data have become available and this has allowed us to study also the role of the $S_{11}(1535), D_{15}(1675), F_{15}(1680)$, $D_{13}(1700), F_{15}(2000)$, and $P_{11}(1900)$. Not all of these resonances are identified to play a considerable role. The results of this analysis of the $p\left(\gamma, K^{+}\right) \Lambda$ data will constitute the basis of the RPR-2011 model.

The resonance parameters are tuned against the $p\left(\gamma, K^{+}\right) \Lambda$ data. For some observables (like the photon asymmetries for example) one finds that the background describes the 


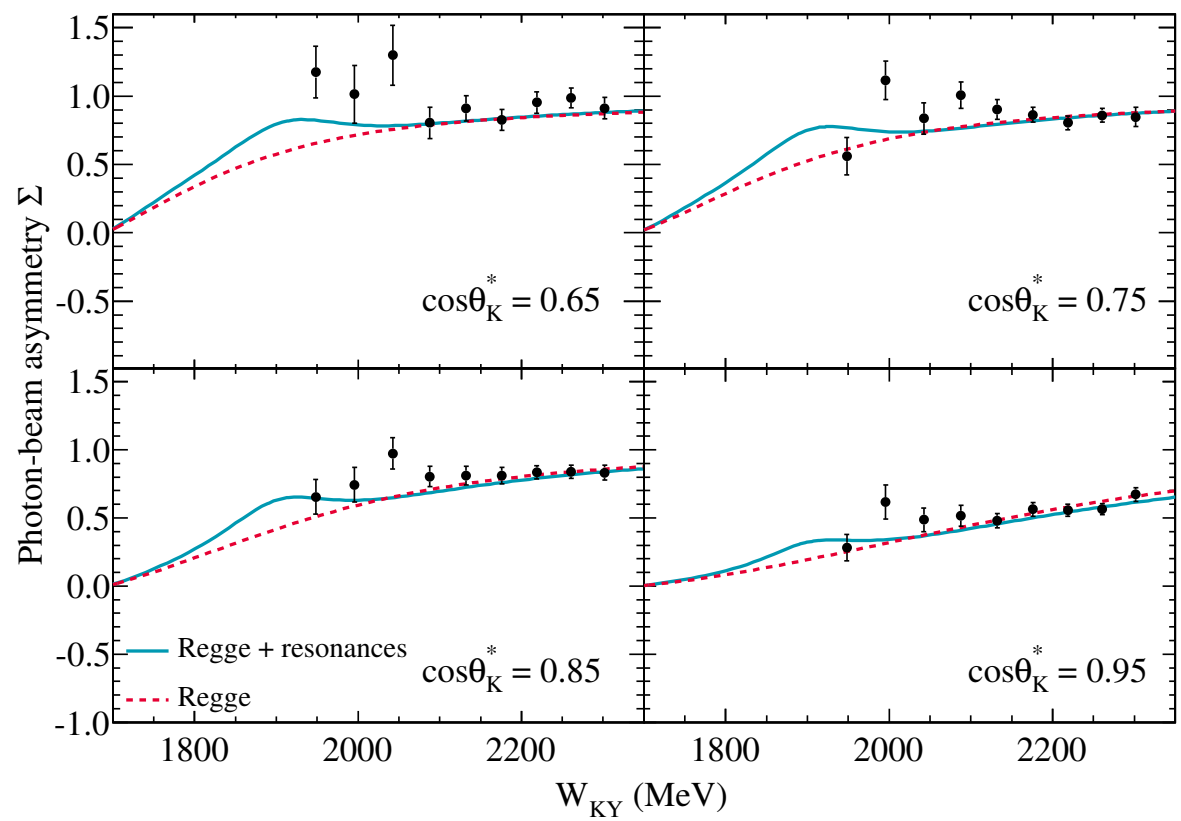

FIGURE 2. The $n\left(\gamma, K^{+}\right) \Sigma^{-}$photon asymmetries as a function of the kaon-hyperon invariant mass $W_{K Y}$ for various $\cos \theta_{K}^{*}$. The dashed lines show the computed contribution from the background diagrams. The solid lines include the background and resonant contributions. The data are from [8]. The error bars represent the statistical errors. Systematic errors amount to $|\Delta \Sigma| \approx 0.2$.

gross feature of the data. We adopt the view that the $p\left(e, e^{\prime} K^{+}\right) \Lambda$ data provide a stringent test of the predictive power of the RPR model. A comparison between the RPR-2007 predictions and recent $p\left(e, e^{\prime} K^{+}\right) \Lambda$ data is shown in Figure 1. The measured longitudinal and transverse cross section are of equal magnitude and exhibit a flat energy dependence. It is observed that the three-parameter background provides a fair estimate of the data. The resonances add some structure and some additional strength and improve the overall quality of agreement with the data.

Strangeness photoproduction involves two $\Lambda\left(p\left(\gamma, K^{+}\right) \Lambda, n\left(\gamma, K^{0}\right) \Lambda\right)$ and four $\Sigma$ $\left(p\left(\gamma, K^{+}\right) \Sigma^{0}, p\left(\gamma, K^{0}\right) \Sigma^{+}, n\left(\gamma, K^{0}\right) \Sigma^{0}, n\left(\gamma, K^{+}\right) \Sigma^{-}\right)$channels. In order to connect the various channels, it suffices to convert the coupling constants featuring in the RPR interaction Lagrangians. In the strong interaction vertices one can fall back on isospin symmetry to determine the conversion factors for the coupling strengths between the different channels. In the electromagnetic interaction vertices in the $s$ channel the coupling strengths $\kappa_{N^{*} N}$ can be written in terms of the photocoupling helicity amplitudes $\mathscr{A}_{J}^{N}$. For example, after equating the proton and neutron mass, one finds for a $N^{*}$ with $J=\frac{1}{2}$

$$
\frac{\kappa_{N^{*} n}}{\kappa_{N^{*} p}}=\frac{\mathscr{A}_{\frac{1}{2}}^{n}}{\mathscr{A}_{\frac{1}{2}}^{p}} .
$$

Experimentally determined values for the helicity amplitudes are available from analyses like SAID [9] or RPP [10] of the pion production data. Unfortunately, in the second and higher resonance region relevant to kaon photoproduction the extracted values for 
$\mathscr{A}_{J}^{N}$ have considerable error bars and are only available for some selected resonances. In addition, the various analyses often produce inconsistent results. In Ref. [11] we have addressed the issue whether the RPR model can predict $n\left(\gamma, K^{+}\right) \Sigma^{-}$observables starting from a limited set of parameters which are constrained against the $p\left(\gamma, K^{+}\right) \Sigma^{0}$ reaction channel. In Figure 2 we show the computed RPR result for the $n\left(\gamma, K^{+}\right) \Sigma^{-}$photon asymmetries. The data result from selecting quasi-free events in $d\left(\gamma, K^{+} \Sigma^{-}\right) p$ measurements. The model calculations include both the background and the resonant contribution and represent predictions which are anchored to the $p\left(\gamma, K^{+}\right) \Sigma^{0}$ channel through isospin symmetry. It is remarkable that with the background diagrams one obtains a fair description of the angular and energy dependence of the photon asymmetries. One can conclude that RPR framework allows one to determine the background contributions to $N(\gamma, K) Y$ in a highly resilient fashion. The resonance contributions to the $n\left(\gamma, K^{+}\right) \Sigma^{-}$ photon asymmetries are rather moderate. In Ref. [11] it is shown that the resonances represent large corrections to the differential cross sections. With regard to the resonance contributions, the mentioned uncertainties on the helicity amplitudes heavily restrain the predictive power of the RPR model and other isobar models for $n(\gamma, K) Y$. The error bars on the helicity amplitudes, for example, give rise to sizable theoretical error bands in the computed resonance contributions [11].

The RPR model provides the elementary production operator for a recently developed covariant model for $d(\gamma, K Y) N$ reactions [12]. The role of $Y N$ final-state interactions (FSI) has been evaluated. At low missing momenta, the effect is small and the deuteron acts as a real neutron target. At high missing momenta, the $Y N$ FSI are large. Under those conditions, the $d(\gamma, K Y) N$ reaction provides a good window on the elusive $Y N$ interaction. At high missing momenta, however, the cross sections are small and the computed $d(\gamma, K Y) N$ observables are prone to uncertainties stemming from the deuteron wave function and the off-shell extrapolation of the elementary production operator.

\section{REFERENCES}

1. T. Corthals, J. Ryckebusch, and T. Van Cauteren, Phys.Rev. C73, 045207 (2006), nucl-th/ 0510056.

2. T. Corthals, D. G. Ireland, T. Van Cauteren, and J. Ryckebusch, Phys.Rev. C75, 045204 (2007), nucl-th/0612085.

3. M. Coman, et al., Phys.Rev. C81, 052201(R) (2010), 0911.3943.

4. L. De Cruz, D. G. Ireland, P. Vancraeyveld, and J. Ryckebusch, Phys.Lett. B694, 33 (2010), 1004. 0353.

5. M. Guidal, J. M. Laget, and M. Vanderhaeghen, Nucl.Phys. A627, 645 (1997).

6. B. Dey, and C. A. Meyer (2011), 1106.0479.

7. M. McCracken, et al., Phys.Rev. C81, 025201 (2010), 0912.4274.

8. H. Kohri, et al., Phys.Rev.Lett 97, 082003 (2006), hep-ex/ 0602015.

9. R. A. Arndt, I. I. Strakovsky, and R. L. Workman, Phys.Rev. C53, 430 (1996), nucl-th/ 9509005.

10. C. Amsler, et al., Phys.Lett. B667, 1 (2008).

11. P. Vancraeyveld, L. De Cruz, J. Ryckebusch, and T. Van Cauteren, Phys.Lett. B681, 428 (2009), 0908.0446.

12. P. Vancraeyveld, L. De Cruz, J. Ryckebusch, and T. Van Cauteren, EPJ Web Conf. 3, 03013 (2010), 0912.2679. 